\title{
Hybrid TODIM Method with Crisp Number and Probability Linguistic Term Set for Urban Epidemic Situation Evaluation
}

\author{
Wenshuai Wu (iD) and Zeshui Xu $\mathbb{D D}^{2}$ \\ ${ }^{1}$ Guangdong Academy of Decision Sciences, Sun Yat-sen University, Guangzhou, Guangdong 510275, China \\ ${ }^{2}$ Business School, Sichuan University, Chengdu, Sichuan 610065, China \\ Correspondence should be addressed to Wenshuai Wu; wuwsh8@mail.sysu.edu.cn
}

Received 7 April 2020; Accepted 10 June 2020; Published 19 August 2020

Academic Editor: Átila Bueno

Copyright (c) 2020 Wenshuai Wu and Zeshui Xu. This is an open access article distributed under the Creative Commons Attribution License, which permits unrestricted use, distribution, and reproduction in any medium, provided the original work is properly cited.

Emergency medical services during the COVID-19 epidemic have become the focus of worldwide attention, and how to effectively respond to urban epidemic situation during a complex environment has become a global challenge. Emergency decision-making can be considered as a multicriteria decision-making (MCDM) problem, which involves multiple criteria or attributes about qualitative and quantitative aspects. So, in this paper, based on the TODIM method, a hybrid TODIM method with crisp number and probability linguistic term set is first provided to evaluate the severity of urban COVID-19 epidemic situation during a complex humanitarian crisis environment. In this hybrid method, the quantitative aspects are evaluated on the basis of precise numerical values, and the qualitative aspects are evaluated by means of probability linguistic term set, which can not only express their judgments or linguistic preference with multiple linguistic terms but also reflect different importance degrees or probability degrees of all the possible linguistic information or preference information. In addition, the concept of entropy and probability linguistic entropy is applied to induce hybrid criteria weight information. Furthermore, sensitivity analysis of the parameter about attenuation factor of the losses in the hybrid TODIM method, which considers the psychology factors and cognitive behavior of the DMs, is further conducted on a case study, to verify the effectiveness and stability of the proposed method for urban epidemic situation evaluation according to the results of this study.

\section{Introduction}

The frequent occurrence of emergency medical incidents has caused a great deal of property losses and a lot of casualties [1]. Until April 5, 2020, the global COVID-19 epidemic has gotten further intensified as the global death toll due to this deadly virus soars to 64,701 , and the total confirmed cases surge to 1,201,591 around the world according to the statistical data provided by Johns Hopkins University. The highest number of confirmed cases in the world is in the United States, with a cumulative total of more than 310,000 cases, and the highest number of deaths is in Italy, with a cumulative total of 15,362 cases. Emergency medical services during the COVID-19 epidemic have become the focus of worldwide attention, and the response to the COVID-19 outbreak has become a global challenging issue.
Emergency medical management and epidemic situation evaluation have a complex evolutionary process, and emergency decision-making is very difficult to deal with the traditional decision theory. The decision-making on epidemic situation evaluation of accidental medical emergencies usually concerns about multiple criteria or attributes about qualitative and quantitative aspects, a finite number of alternatives, semantic benefits, and interests of multiple stakeholders, and it can be considered as a complex multicriteria decision-making (MCDM) problem. Kahneman and Tversky [2] pointed out that the decision-makers (DMs) play a limited rational role in the complex decision-making process; in other words, the psychology and behavior of DMs may be the important factors which can affect the decision-making results. The TODIM method, first proposed by Gomes and Lima [3], is an interactive MCDM method considering the psychology factors and cognitive behavior of the DMs [4]. Liu and Teng [5] proposed 
an extended TODIM method based on intuitionistic uncertain linguistic variables for multiple attribute group decisionmaking. Rani et al. [6] proposed an extended intuitionistic fuzzy TODIM technique for multicriteria decision-making method based on Shapley weighted divergence measure. Davoudabadi et al. [7] proposed a new last aggregation method of multiattribute group decision-making based on concepts of TODIM, WASPAS, and TOPSIS under intervalvalued intuitionistic fuzzy uncertainty. Although this TODIM method has done a lot of outreach and extension, it is still not well studied and applied for epidemic situation evaluation in the emergency medical decision-making process.

The DMs usually need data and information to make effective decisions, especially for complex decisions. In general, emergency decision-making on the COVID-19 outbreak is considered as a multicriteria decision-making (MCDM) problem, which involves multiple criteria or attributes about qualitative and quantitative aspects. The quantitative aspects are evaluated on the basis of precise numerical values, and the qualitative aspects are evaluated by means of uncertainty including vagueness and ambiguity [8], such as fuzzy set theory, rough set theory, and neutrosophic set theory. However, vague data are uncertain because they lack detail or precision, and ambiguous data are uncertain because they are subject to multiple interpretations [9]. Probability linguistic term set (PLTS) is provided by Pang et al. [10] as a new type of linguistic variable, which can not only express their judgments or linguistic preference with multiple linguistic terms but also reflect different importance degrees or probability of all the possible linguistic information or preference information. Liu and You [4] extended the TODIM with PLTS to solve the multiattribute decision-making problems by an example of corporate project evaluation, and the objective weights are determined based on the traditional measure of information entropy under probabilistic linguistic environment. In this paper, firstly, the extended hybrid TODIM method with crisp number and PLTS is first provided to evaluate the severity of urban COVID-19 epidemic situation. Secondly, the objective weights for PLTS are induced and determined based on the concept of probability linguistic entropy, which has the advantages of simple calculation and clear concept.

In emergency medical management, decision-making process has become more and more complex because many of the attributes are difficult to quantify [11-13] and decisions are usually made with subjective preference information in human perceptual timeframes under pressure, lack of knowledge, and data cases [14, 15]. Furthermore, their effectiveness can be constrained due to the complexity of the decision-making process and the intricacy of systems $[13,16]$. How to effectively respond to the accidental medical emergencies and evaluate the severity of urban COVID-19 epidemic situation during a complex environment has become a global challenge.

To sum up, this paper focuses on the development of a hybrid TODIM method with crisp number and probability linguistic term set, based on the TODIM method which can consider the psychology factors and cognitive behavior of the DMs, for aiming to assist DMs to evaluate the severity of urban COVID-19 epidemic situation involved in emergency medical management. Five main works of this paper that have led to its novelty are as follows: (1) A hybrid TODIM method with crisp number and probability linguistic term set is first provided to evaluate the severity of urban COVID-19 epidemic situation during a complex humanitarian crisis environment. (2) The proposed hybrid TODIM method involves multiple criteria or attributes about qualitative and quantitative aspects, considering the complexity of emergency decision-making process. (3) The proposed hybrid TODIM method can not only express their judgments or linguistic preference with multiple linguistic terms, but also reflect different importance degrees or probability degrees of all the possible linguistic information or preference information. (4) The concept of entropy and probability linguistic entropy is applied to induce hybrid criteria weight information, which has the advantages of simple calculation and clear concept. (5) Sensitivity analysis of the parameter in the hybrid TODIM method, which considers the psychology factors and cognitive behavior of the DMs, is further carried out to demonstrate the effectiveness and stability of the proposed hybrid method for urban epidemic situation evaluation.

The rest of this paper is organized as follows. Section 2 reviews the related research. Section 3 introduces some brief backgrounds required for this paper. In Section 4, a hybrid TODIM method with crisp number and PLTS is proposed. In Section 5, an empirical study on urban COVID-19 epidemic situation evaluation during a complex humanitarian crisis environment is conducted to verify our provided method. Finally, Section 6 concludes the paper.

\section{Related Works}

The frequent occurrence of emergency medical incidents has caused a great deal of property losses and a lot of casualties [17]. In the meantime, it has also promoted the theoretical research and technology development to deal with emergency problems. Pullum and Ramanathan [18] indicated that datadriven machine learning tools should be developed to automate discovery for helping DMs gain quantifiable insights on emerging disease. Wang et al. [19] identified EMS emergency responses with attempted advanced airway management. Temple et al. [20] employed the GRADE method to conduct a systematic literature review and search multiple databases to examine the effects of caffeine on results of interest. Lazzara et al. [21] synthesized the literature pertinent to multiteam systems and emergency medical services to assist the prehospital community in strengthening patient care. However, epidemic situation evaluation has a complex evolutionary process. The emergency decision-making on epidemic situation evaluation usually concerns about multiple criteria or attributes about qualitative and quantitative aspects, a finite number of alternatives, semantic benefits, and interests of multiple stakeholders, which is essentially considered as a complex multicriteria decision-making (MCDM) problem. Based on the complexity of the emergency decisionmaking process, the TODIM method is still not well studied and applied for epidemic situation evaluation in the emergency medical decision-making, although it has done a lot of outreach and extension. 
MCDM has made remarkable progress and has evolved into a mature discipline, which concerns about multiple conflicting criteria, a finite number of alternatives, and multiple opinions of DMs [1, 22-24]. Kahneman and Tversky [2] pointed out that the DMs play a limited rational role in the complex decision-making process; in other words, the psychology and behavior of DMs may be the important factors which affect the decision-making results. TODIM method, an interactive MCDM method, is proposed by Gomes and Lima [3], which can consider the psychology factors and cognitive behavior of the DMs [4] to address decision-making problems [10, 25-27].

Decisions usually need data information, particularly for complex decisions, which involve multiple criteria about qualitative and quantitative aspects. The quantitative aspects are usually evaluated on the base of accurate numerical values, and the qualitative aspects are usually evaluated by means of uncertainty including vagueness and ambiguity [8]. However, vague data are uncertain because they lack detail or precision, and ambiguous data are uncertain because they are subject to multiple interpretations [9]. It is impossible to deal with this imprecise situation with the traditional MCDM method. PLTS is provided by Pang et al. [10] as a new type of linguistic variable for indicating fuzzy data information, which can express and reflect different importance degrees or probability of all the possible linguistic information with multiple linguistic terms. For instance, when one DM is evaluating the severity of the urban epidemic situation, based on self-awareness of the epidemic, the DM may think he/she is $30 \%$ sure the urban epidemic is "very serious," 50\% sure it is "serious," and 20\% sure it is "general". It is obvious that the problem of urban epidemic situation evaluation needs to be integrated with PTLS to express complex and uncertain information. So, this paper, based on the TODIM method, provides a hybrid TODIM method with crisp number and PLTS to evaluate the severity of urban COVID-19 epidemic situation.

Besides, in the application of TODIM method, some scholars have done some research and development. Fan et al. [28] proposed an extended TODIM method to solve the hybrid MADM problem with crisp numbers, interval numbers, and fuzzy numbers. Liu and Teng [29] extended the TODIM method to 2-dimensional uncertain linguistic information for multiple attribute group decision-making. Liu and You [4] extended the TODIM with PLTS to solve the multiattribute decision-making problems according to an example of corporate project evaluation, and during the probabilistic linguistic environment, the objective weights are determined based on the traditional measure of information entropy. Wei [26] expanded the TODIM model to the MADM with the picture fuzzy numbers. Although some achievements have been made in the application of TODIM method, the hybrid TODIM method with crisp number and PLTS has not been fully studied. Furthermore, in this paper, the extended hybrid TODIM method is first proposed to evaluate the severity of urban COVID-19 epidemic situation. In addition, Zhang et al. [27] explored the water security evaluation based on the TODIM method with probabilistic linguistic term sets, where a programming model is developed to derive the attribute weights. The attribute weights determined by mathematical programming may be separated from the characteristics of the data itself to some extent. So, in this paper, the concept of entropy and probability linguistic entropy is applied to induce hybrid criteria weight information, which has the advantages of simple calculation and clear concept.

In view of the foregoing, this paper based on the TODIM method provides a hybrid TODIM method with crisp number and PLTS to evaluate the severity of urban COVID19 epidemic situation for aiding DMs involved in emergency medical management during a complex humanitarian crisis environment.

\section{Preliminaries}

In this section, some brief backgrounds of PLTS, the comparison between PLTSs, the normalization of PLTS, and the TODIM method are introduced separately.

\subsection{Probabilistic Linguistic Term Set}

Definition 1 (see [10]). Based on the additive linguistic term set $S=\left\{s_{\alpha}=-\tau, \ldots,-1,0, \ldots, \tau\right\}[30,31]$, the probabilistic linguistic term set (PLTS) can be defined by Pang et al. [10] as follows:

$$
L(p)=\left\{L^{(k)}\left(p^{k}\right) \mid L^{(k)} \in S, p^{(k)} \geq 0, k=1,2, \ldots, \# L(p), \sum_{k=1}^{\# L(p)} p^{(k)} \leq 1\right\}
$$

where $L^{(k)}\left(p^{(k)}\right)$ represents the linguistic term $L^{(k)}$ associated with the probability $p^{(k)}$ and $\# L(p)$ is the number of all different linguistic terms in $L^{(p)}$.

If $\sum_{k=1}^{\# L(p)} p^{(k)}=1$, then the PLTSs have complete probabilistic information of all possible linguistic terms if $\sum_{k=1}^{\# L(p)} p^{(k)}<1$, then the PLTSs have partial probabilistic information; if $\sum_{k=1}^{\# L(p)} p^{(k)}=0$, then the
PLTSs have completely unknown probabilistic information.

Definition 2 (see [27]). Given a probabilistic linguistic term set $L(p)=\left\{L^{(k)}\left(p^{k}\right) \mid k=1,2, \ldots, \# L(p), \sum_{k=1}^{\# L(p)} p^{(k)} \leq 1\right\}$, on the additive linguistic term set $S=\left\{s_{\alpha}=-\tau, \ldots\right.$, $-1,0, \ldots, \tau\}$, the negation operation for $L(p)$ is given by 


$$
\operatorname{neg}(L(p))=\left\{\widehat{L}^{(k)}\left(p^{(k)}\right) \mid \widehat{L}^{(k)}=\operatorname{neg}\left(L^{(k)}\right)=\operatorname{neg}\left(S_{\alpha}\right), \quad k=1,2, \ldots, \# L(p)\right\}
$$

where neg $(L(p))$ is the negation operation for linguistic terms [31], defined as $\operatorname{neg}\left(S_{\alpha}\right)=S_{-\alpha}$, especially, $\operatorname{neg}\left(S_{0}\right)=S_{0}$.

3.2. The Comparison between PLTSs. First, the score of PLTS is defined and introduced by Pang et al. [10] as follows.

Definition 3 (see [32]). Let $L(p)=\left\{L^{(k)}\left(p^{(k)}\right) \mid k=\right.$ $1,2, \ldots, \# L(p)\}$ be a PLTS and $r^{(k)}$ be the subscript of the $L^{(k)}$, then the score of $L(p)$ is given by

$$
E(L(p))=s_{\bar{\alpha}}
$$

where $\bar{\alpha}=\sum_{k=1}^{\# L(p)} r^{(k)} p^{(k)} / \sum_{k=1}^{\# L(p)} p^{(k)}$.

For any two PLTSs $L_{1}(p)$ and $L_{2}(p)$, if $E\left(\left(L_{1}(p)\right)>E\left(L_{2}(p)\right)\right)$, then $L_{1}(p)$ is superior to $L_{2}(p)$, denoted by $L_{1}(p)>L_{2}(p)$; if $E\left(\left(L_{1}(p)\right)<E\left(L_{2}(p)\right)\right)$, then $L_{2}(p)$ is superior to $L_{1}(p)$, denoted by $L_{1}(p)<L_{2}(p)$; if $E\left(\left(L_{1}(p)\right)=E\left(L_{2}(p)\right)\right)$, then these two PLTSs cannot be distinguished. In this case, the deviation degree of a PLTS is further defined by Pang et al. [10] as follows.

Definition 4 (see [10]). Let $L(p)=\left\{L^{(k)}\left(p^{(k)}\right) \mid k=\right.$ $1,2, \ldots, \# L(p)\}$ be a PLTS and $r^{(k)}$ be the subscript of the $L^{(k)}$, and $E(L(p))=s_{\bar{\alpha}}$, then the deviation degree of $L(p)$ is given by

$$
\sigma(L(p))=\left(\frac{\left.\sum_{k=1}^{\# L(p)} p^{(k)}\left(r^{(k)}-\bar{\alpha}\right)^{2}\right)^{1 / 2}}{\sum_{k=1}^{\# L(p)} p^{(k)} .}\right.
$$

For two PLTSs $L_{1}(p)$ and $L_{2}(p)$ with $E\left(L_{1}(p)\right)=E\left(L_{2}(p)\right)$, if $\sigma\left(L_{1}(p)\right)>\sigma\left(L_{2}(p)\right)$, then $L_{1}(p)<L_{2}(p)$; if $\sigma\left(L_{1}(p)\right)=\sigma\left(L_{1}(p)\right)$, then $L_{1}(p)$ is indifferent to $L_{2}(p)$, denoted by $L_{1}(p) \sim L_{2}(p)$.

Definition 5 (see [10]). Let $L_{1}(p)=\left\{L_{1}^{k}\left(p^{(k)}\right) \mid k=\right.$ $\left.1,2, \ldots, \# L_{1}(p)\right\}$ and $L_{2}(p)=\left\{L_{2}^{k}\left(p^{(k)}\right) \mid k=1,2, \ldots, \# L_{2}\right.$ $(p)\}$ be any two PLTSs, $\# L_{1}(p)=\# L_{2}(p)$, the distance between $L_{1}(p)$ and $L_{2}(p)$ can be defined as follows:

$$
d\left(L_{1}(p), L_{1}(p)\right)=\sqrt{\frac{\sum_{k=1}^{\# L_{1}(p)}\left(p_{1}^{(k)} r_{1}^{(k)}-p_{2}^{(k)} r_{2}^{(k)}\right)^{2}}{\# L_{1}(p)}} .
$$

3.3. The Normalization of PLTS. The normalization of PLTSs usually has two distinct tasks. One is to estimate the ignorance degree of probabilistic information, and the other is to normalize the cardinality for computational convenience.

Definition 6 (see [10]). Given a PLTS $L(p)$ with $\sum_{k=1}^{\# L(p)}$ $p^{(k)}<1$, then the normalized PLTS $\widetilde{L}(p)$ is defined as follows:

$$
\widetilde{L}(p)=\left\{L^{(k)}\left(p^{(k)}\right) \mid k=1,2, \ldots, \# L(p)\right\},
$$

where $\tilde{p}^{(k)}=p^{(k)} / \sum_{k=1}^{\# L(p)} p^{(k)}$, for all $k=1,2, \ldots, \# L(p)$.

Let $L_{1}(p)=\left\{L_{1}^{(k)}\left(p_{1}^{(k)}\right) \mid k=1,2, \ldots, \# L(p)\right\} \quad$ and $L_{2}(p)=\left\{L_{2}^{(k)}\left(p_{2}^{(k)}\right) \mid k=1,2, \ldots, \# L(p)\right\}$, then the normalization process can be obtained as follows:

(1) If $\sum_{k=1}^{\# L(p)} p^{(k)}<1$, then according to formula (6), $\widetilde{L}(p)$ can be calculated.

(2) If $\# L_{1}(p) \neq \# L_{2}(p)$, then some elements can be added to the one with the smaller number of elements. That is to say, if $\# L_{1}(p)>\# L_{2}(p)$, then the $\# L_{1}(p)-\# L_{2}(p)$ linguistic terms need to be added to $L_{2}(p)$, which make the numbers of all different linguistic terms in $L_{1}(p)$ and $L_{2}(p)$ equal. The added linguistic terms should be the smallest ones in $L_{2}(p)$, and their probabilities should be zero.

3.4. The TODIM Method. The TODIM method, proposed by Gomes and Lima [3], is an interactive MCDM method, which can capture psychology factors and cognitive behavior of DMs. On the base of prospect theory [2], the alternatives are ranked according to their overall dominance degree based on the dominance degree of each alternative over others with respect to each criterion. The steps of TODIM method can be summarized as follows [3].

Suppose there are $A_{i}(i=1,2, \ldots, m)$ alternatives and $C_{j}(j=1,2, \ldots, n)$ criteria.

Step 1: identify the decision matrix $X=\left(x_{i j}\right)_{m \times n}$, where $x_{i j}(i=1,2, \ldots, m, j=1,2, \ldots, n)$ is the $j$ th criteria value with respect to the $i$ th alternative, and $x_{i j}$ are all crisp numbers.

Step 2: obtain the normalized decision matrix $G=\left(g_{i j}\right)_{m \times n}$. The normalized decision matrix can be obtained by using the normalization method.

Step 3: calculate the relative weight $w_{j r}$ of the criteria $C_{j}$ to the reference criteria $C_{r}$ as follows:

$$
w_{j r}=\frac{w_{j}}{w_{r}}(j=1,2, \ldots, n),
$$

where $w_{j}$ is the weight of the criteria $C_{j}$ and $w_{r}=\max \left\{w_{j} \mid j \in 1,2, \ldots, n\right\}$.

Step 4: obtain the dominance degree of alternative $A_{i}$ over the alternative $A_{t}$, as follows:

$$
\mathcal{\vartheta}\left(A_{i}, A_{t}\right)=\sum_{j=1}^{n} \phi_{j}\left(A_{i}, A_{t}\right), \quad \forall(i, t) .
$$

where $\phi_{j}\left(A_{i}, A_{t}\right)$ is the dominance degree of the alternative $A_{i}$ over the alternative $A_{t}$ with respect to the criterion $C_{j}$, and it can be calculated as follows: 


$$
\phi_{j}\left(A_{i}, A_{t}\right)= \begin{cases}\sqrt{\frac{w_{j r}\left(g_{i j}-g_{t j}\right)}{\sum_{j=1}^{n} w_{j r}},} & \text { if } g_{i j}-g_{t j}>0, \\ 0, & \text { if } g_{i j}-g_{t j}=0, \\ -\frac{1}{\theta} \sqrt{\frac{\sum_{j=1}^{n} w_{j r}\left(g_{i j}-g_{t j}\right)}{w_{j r}},} & \text { if } g_{i j}-g_{t j}<0,\end{cases}
$$

where the parameter $\theta$ is the attenuation factor of the losses. There are three cases: (1) if $g_{i j}-g_{t j}>0$, then $\phi_{j}\left(A_{i}, A_{t}\right)$ represents a gain; (2) if $g_{i j}-g_{t j}=0$, then $\phi_{j}\left(A_{i}, A_{t}\right)$ represents a nil; and (3) if $g_{i j}-g_{t j}<0$, then $\phi_{j}\left(A_{i}, A_{t}\right)$ represents a loss.

Step 5: get the overall dominance degree of the alternative $A_{i}$ as follows:

$$
\delta\left(A_{i}\right)=\frac{\sum_{t=1}^{m} \vartheta\left(A_{i}, A_{t}\right)-\min _{i}\left\{\sum_{t=1}^{m} \vartheta\left(A_{i}, A_{t}\right)\right\}}{\max _{i}\left\{\sum_{t=1}^{m} \vartheta\left(A_{i}, A_{t}\right)\right\}-\min _{i}\left\{\sum_{t=1}^{m} \vartheta\left(A_{i}, A_{t}\right)\right\}} .
$$

Step 6: sort the alternatives according to the overall dominance degree $\delta\left(A_{i}\right)$.

The larger the value of $\delta\left(A_{i}\right)$ is, the better the alternative is.

\section{Hybrid TODIM Method}

In this section, a hybrid TODIM method with crisp number and PLTS is provided in detail.

For an MCDM problem, suppose that there are $A_{i}(i=$ $1,2, \ldots, m)$ alternatives and $C_{j}(j=1,2, \ldots, n)$ criteria. Based on the additive linguistic term set $S=\left\{S_{\alpha}\right.$ $\mid \alpha=-\tau, \ldots,-1,0,1, \ldots, \tau\}$, the DMs assess the alternatives $A_{i}$ for the criteria $C_{j}$, and the evaluation results during the probabilistic linguistic terms environment are given by $L(p)=\left\{L^{(k)}\left(p^{(k)}\right) \mid k=1,2, \ldots, \# L(p), \sum_{k=1}^{\# L(p)} p^{(k)} \leq 1\right\}$.

The specific steps of the hybrid TODIM method with crisp number and PLTS are presented as follows:

Step 1: identify the decision matrix $X=\left(x_{i j}\right)_{m \times n}$. The initial decision matrix can be constructed and identified with crisp number $a_{i j}$ and PLTS $L_{i j}(p)$.

Step 2: obtain the normalized decision matrix $G=\left(g_{i j}\right)_{m \times n}$. The normalized decision matrix can be obtained as follows:

(1) Crisp number: the normalization of crisp number $\widetilde{a_{i j}}$ can be obtained as follows:

$$
\begin{aligned}
& \widetilde{a_{i j}}=\frac{a_{i j}}{\max _{j} a_{i j}}, \quad \text { for benefit criteria, } \\
& \widetilde{a_{i j}}=\frac{\min _{j} a_{i j}}{a_{i j}}, \quad \text { forcostcriteria. }
\end{aligned}
$$

(2) PLTS: the normalization of PLTS $L_{i j}(p)$ can be obtained as follows:

$\widetilde{L_{i j}}\left(p_{i j}^{(k)}\right)= \begin{cases}L_{i j}\left(p_{i j}^{(k)}\right), & \text { for benefit criteria, } \\ \operatorname{neg}\left(L_{i j}\left(p_{i j}^{(k)}\right)\right), & \text { forcostcriteria, }\end{cases}$

where $\operatorname{neg}\left(L_{i j}\left(p_{i j}^{(k)}\right)\right)$ is the negation operation for $L_{i j}\left(p_{i j}^{(k)}\right)$, which is defined in Definition 2. Besides, each criteria value of PLTS should be normalized according to Definition 6.

Step 3: calculate the entropy weight for crisp number and probability linguistic entropy weight for PLTS. There are two steps:

(1) Crisp number: the entropy weight for crisp number can be determined as follows:

(a) Obtain the entropy: according to the definition of entropy, entropy $E_{j}$ of criteria $C_{j}$ is determined as follows:

$$
E_{j}=-\frac{1}{\ln m} \sum_{i=1}^{m} a_{i j} \ln a_{i j} .
$$

(b) Obtain the entropy weight $w_{j}^{\prime}$ of criteria $C_{j}$ :

$$
w_{j}^{\prime}=\frac{1-E_{j}}{n-\sum_{j=1}^{n} E_{j}} \quad j=1,2, \ldots, n .
$$

(c) Normalize the entropy weight $w_{j}$ : the entropy weight can be normalized as follows:

$$
w_{j}=\frac{w_{j}^{\prime}}{\sum_{j=1}^{n} w_{j}^{\prime}}, \quad j=1,2, \ldots, n .
$$

(2) PLTS: based on the concepts of entropy, the probability linguistic entropy weight for PLTS can be obtained as follows:

(a) Based on the additive linguistic term set $S=\left\{s_{\alpha}=-\tau, \ldots,-1,0,1 \ldots, \tau\right\}[30,31], \quad$ six methods for calculating the entropy value of a single linguistic term are proposed by Farhadinia [32]. One of the methods is as follows:

$$
E\left(s_{\alpha}\right)=\sin \frac{\pi}{2}\left(\frac{\alpha+\tau}{2 \tau}\right)+\sin \frac{\pi}{2}\left(\frac{-\alpha+\tau}{2 \tau}\right)-1 .
$$

(b) Let $L(p)=\left\{L^{(k)}\left(p^{(k)}\right) \mid k=1,2, \ldots, \# L(p)\right\}$ be a PLTS, the probability linguistic entropy weight can be induced as follows:

$$
E_{i j}\left(L_{i j}\left(p_{i j}^{(k)}\right)\right)=\sum_{i=1}^{\# L(p)} p_{i j}^{(k)}\left[E_{i j}\left(S_{\alpha}\right)\right] .
$$


(c) Normalize the probability linguistic entropy: the probability linguistic entropy can be normalized as follows:

$G_{i j}=\frac{E_{i j}}{\max _{1 \leq i \leq m}\left\{E_{i j}\right\}} \quad i=1,2, \ldots, m, j=1,2, \ldots, n$.

(d) Determine the weight: the weight of each criterion can be determined as follows:

$$
w_{j}^{\prime}=\frac{1-\overline{G_{j}}}{n-\bar{G}} \quad j=1,2, \ldots, n .
$$

where $\overline{G_{j}}=\sum_{i=1}^{m} G_{i j}, \bar{G}=\sum_{i=1}^{m} \sum_{j=1}^{n} G_{i j}$.

(e) Determine the normalized weight: the normalized weight can be determined as follows:

$$
w_{j}=\frac{w_{j}^{\prime}}{\sum_{j=1}^{n} w_{j}^{\prime}}, \quad j=1,2, \ldots, n .
$$

Step 4: calculate the relative weight $w_{j r}$ of the criteria $C_{j}$ to the reference criteria $C_{r}$ as follows:

$$
w_{j r}=\frac{w_{j}}{w_{r}}(j=1,2, \ldots, n),
$$

where $w_{j}$ is the entropy weight for crisp number, or the probability linguistic entropy weight for PLTS of the criteria $C_{j}$ and $w_{r}=\max \left\{w_{j} \mid j \in 1,2, \ldots, n\right\}$.

Step 5: obtain the dominance degree $\vartheta$ of alternative $A_{i}$ over the alternative $A_{t}$, as follows:

$\vartheta\left(A_{i}, A_{t}\right)=\sum_{j=1}^{n} \phi_{j}\left(A_{i}, A_{t}\right) \quad(i, t=1,2, \ldots, \mathrm{m})$,

where $\phi_{j}\left(A_{i}, A_{t}\right)$ is the dominance degree of the alternative $A_{i}$ over the alternative $A_{t}$ with respect to the criterion $C_{j}$, and it can be calculated as follows:

$$
\phi_{j}\left(A_{i}, A_{t}\right)= \begin{cases}\sqrt{\frac{\left.w_{j r} d\left(\widetilde{L_{i j}}\left(p_{i j}\right) t-n \widetilde{L_{i j}} q\left(p_{t j}\right)\right)\right)}{\sum_{j=1}^{n} w_{j r}},} & \text { if } \widetilde{L_{i j}}\left(p_{i j}\right)>\widetilde{L_{i j}}\left(p_{t j}\right), \\ 0, & \text { if } \widetilde{L_{i j}}\left(p_{i j}\right) \sim \widetilde{L_{i j}}\left(p_{t j}\right), \\ -\frac{1}{\theta} \sqrt{\frac{\sum_{j=1}^{n} w_{j r} d\left(\left(\widetilde{L_{i j}}\left(p_{i j}\right) t-n \widetilde{L_{i j}} q\left(p_{t j}\right)\right)\right)}{w_{j r}},} & \text { if } \widetilde{L_{i j}}\left(p_{i j}\right)<\widetilde{L_{i j}}\left(p_{t j}\right),\end{cases}
$$

where the parameter $\theta$ is the attenuation factor of the losses. There are three cases:

(1) If $\widetilde{L_{i j}}\left(p_{i j}\right) \succ \widetilde{L_{t j}}\left(p_{t j}\right)$, then $\phi_{j}\left(A_{i}, A_{t}\right)$ represents a gain

(2) If $\widetilde{L_{i j}}\left(p_{i j}\right) \sim \widetilde{L_{t j}}\left(p_{t j}\right)$, then $\phi_{j}\left(A_{i}, A_{t}\right)$ represents a nil

(3) If $\widetilde{L_{i j}}\left(p_{i j}\right)<\widetilde{L_{t j}}\left(p_{t j}\right)$, then $\phi_{j}\left(A_{i}, A_{t}\right)$ represents a loss

In this section, the distance measure can be determined as follows:

(1) Crisp number: the distance for crisp number can be determined as follows:

$$
d(a, b)=|a-b| .
$$

(2) PLTS: the distance for PLTS can be determined according to the modified the Hamming distance of PLTSs based on the normalized PLTSs proposed by Lin and $\mathrm{Xu}[33]$.
Step 6: get the overall dominance degree $\delta\left(A_{i}\right)$ of the alternative $A_{i}$ as follows:

$$
\delta\left(A_{i}\right)=\frac{\sum_{t=1}^{m} \vartheta\left(A_{i}, A_{t}\right)-\min _{i}\left\{\sum_{t=1}^{m} \vartheta\left(A_{i}, A_{t}\right)\right\}}{\max _{i}\left\{\sum_{t=1}^{m} \vartheta\left(A_{i}, A_{t}\right)\right\}-\min _{i}\left\{\sum_{t=1}^{m} \vartheta\left(A_{i}, A_{t}\right)\right\}} .
$$

Step 7: sort the alternatives according to the overall dominance degree $\delta\left(A_{i}\right)$. is.

The larger the value of $\delta\left(A_{i}\right)$ is, the better the alternative

\section{A Case Study}

5.1. Problem Description. The outbreak of COVID-19 epidemic has spread rapidly around the world, which has caused a great deal of property losses and a lot of casualties. It is very urgent to evaluate and respond to the urban epidemic situation involved in emergency medical 
management for providing scientific decision basis. Emergency medical management has a complex evolutionary process, and decision-making process has become more and more complex. In this section, a hybrid TODIM method with crisp number and PLTS is provided and presented to evaluate the severity of urban COVID-19 epidemic situation on a case study. The detailed assessment process is as follows.

Five cities $A_{i}(i=1,2,3,4,5)$ around the world and seven criteria $C_{j}(j=1,2, \ldots, 7)$ are selected to illustrate the point. These selected criteria by related literature and expert opinions are as follows: (1) $C_{1}$ : confirmed diagnosis rate per one million population; (2) $\mathrm{C}_{2}$ : cure rate of confirmed cases; (3) $C_{3}$ : mortality rate of confirmed cases; (4) $C_{4}$ : growth rate of confirmed cases in three days; (5) $C_{5}$ : number of beds per capita of confirmed cases; (6) $C_{6}$ : people's awareness of the epidemic; and (7) $C_{7}$ : government's attitude toward the epidemic. Obviously, for evaluating the severity of urban epidemic situation, the criteria $C_{1}, C_{3}$, and $C_{4}$ are of benefit type, and $\mathrm{C}_{2}, \mathrm{C}_{5}, \mathrm{C}_{6}$, and $\mathrm{C}_{7}$ are of cost type. The data information with crisp number and PLTS is presented, as shown in Table 1.

5.2. Empirical Process. Now, the detailed evaluation steps are presented as follows:

Step 1: identify the decision matrix.

The decision matrix with crisp number and PLTS is constructed and identified by the following additive linguistic term set

$$
\begin{gathered}
S=\left(S_{-3}=\text { extremely slight, } S_{-2}=\right.\text { very slight, } \\
S_{-1}=\text { slight, } S_{0}=\text { general, } S_{1}=\text { serious, } \\
\left.S_{2}=\text { very serious, } S_{3}=\text { extremely serious }\right),
\end{gathered}
$$

to assess the severity of urban epidemic situation as shown in Table 1, the data of crisp number originated from the data management platform, a Wechat official account. The data of PLTS come from expert opinion, and the evaluation values during the probabilistic linguistic term environment are obtained and given according to the PLTS: $L(p)=\left\{L^{(k)}\left(p^{k}\right) \mid k=1,2, \ldots\right.$, $\left.\# L(p), \sum_{k=1}^{\# L(p)} p^{(k)} \leq 1\right\}$.

Step 2: obtain the normalized decision matrix. It is obvious that the criteria $C_{1}, C_{3}$, and $C_{4}$ are of benefit type, and $C_{2}, C_{5}, C_{6}$, and $C_{7}$ are of cost type. So the normalization process is relatively simple and the normalized values can be calculated by formulas (11) and (12), Definition 2, and Definition 6, as shown in Table 2.

Step 3: calculate the relative weight. The relative weight is calculated, based on the entropy weight for crisp number and probability linguistic entropy weight for PLTS according to formulas (13)-(21), as shown in Table 3 . And the criteria $C_{1}$ is the reference criteria, which has the greatest weight.

Step 4: obtain the dominance degree $\vartheta$ of the alternative $A_{i}$ over the alternative $A_{t}$ by formulas (22)-(24), based on the dominance degree $\phi_{j}$ of the alternative $A_{i}$ over the alternative $A_{t}$ with respect to the criterion $C_{j}(i, t=1,2, \ldots, 5, j=1,2, \ldots, 7)$ : $\phi_{1}=\left[\begin{array}{ccccc}0.0000 & -1.2671 & -1.5473 & -0.5776 & -0.9357 \\ 0.2753 & 0.0000 & -0.8880 & 0.2450 & 0.1856 \\ 0.3362 & 0.1929 & 0.0000 & 0.3119 & 0.2677 \\ 0.1255 & -1.1278 & -1.4354 & 0.0000 & -0.7362 \\ 0.2033 & -0.8544 & -1.2322 & 0.1599 & 0.0000\end{array}\right]$,

$\phi_{2}=\left[\begin{array}{ccccc}0.0000 & -0.2540 & -0.2694 & -0.2036 & -0.2814 \\ -1.7498 & 0.0000 & 0.0898 & -1.0457 & 0.1211 \\ -1.8560 & -0.6187 & 0.0000 & -1.2150 & 0.0812 \\ -1.4030 & 0.1518 & 0.1764 & 0.0000 & 0.1942 \\ -1.9385 & -0.8342 & -0.5596 & -1.3377 & 0.0000\end{array}\right]$,

$\phi_{3}=\left[\begin{array}{ccccc}0.0000 & 0.2283 & 0.3954 & 0.3954 & 0.2283 \\ -1.0952 & 0.0000 & 0.3228 & 0.3228 & 0.3228 \\ -1.8969 & -1.5488 & 0.0000 & 0.0000 & -1.5488 \\ -1.8969 & -1.5488 & 0.0000 & 0.0000 & -1.5488 \\ -1.0952 & 0.0000 & 0.3228 & 0.3228 & 0.0000\end{array}\right]$,

$\phi_{4}=\left[\begin{array}{ccccc}0.0000 & 0.2054 & 0.2179 & 0.2054 & 0.2445 \\ -2.5395 & 0.0000 & 0.0726 & 0.0000 & 0.1326 \\ -2.6936 & -0.8979 & 0.0000 & -0.8979 & 0.1110 \\ -2.5395 & 0.0000 & 0.0726 & 0.0000 & 0.1326 \\ -3.0226 & -1.6392 & -1.3715 & -1.6392 & 0.0000\end{array}\right]$,

$\phi_{5}=\left[\begin{array}{ccccc}0.0000 & 0.1387 & -1.2804 & -1.7891 & -1.2495 \\ -0.7903 & 0.0000 & -1.5046 & -1.9558 & -1.4785 \\ 0.2247 & 0.2640 & 0.0000 & -1.2495 & 0.0490 \\ 0.3139 & 0.3432 & 0.2193 & 0.0000 & 0.2247 \\ 0.2193 & 0.2594 & -0.2794 & -1.2804 & 0.0000\end{array}\right]$,

$\phi_{6}=\left[\begin{array}{ccccc}0.0000 & 0.0600 & -0.4691 & -0.3831 & -0.6635 \\ -0.7923 & 0.0000 & -0.9208 & -0.8800 & -1.0334 \\ 0.0355 & 0.0697 & 0.0000 & 0.0205 & -0.4691 \\ 0.0290 & 0.0666 & -0.2709 & 0.0000 & -0.5417 \\ 0.0502 & 0.0783 & 0.0355 & 0.0410 & 0.0000\end{array}\right]$,

$\phi_{7}=\left[\begin{array}{ccccc}0.0000 & -0.4785 & -0.4144 & -0.2393 & -0.3384 \\ 0.0464 & 0.0000 & 0.0232 & 0.0402 & 0.0328 \\ 0.0402 & -0.2393 & 0.0000 & 0.0328 & 0.0232 \\ 0.0232 & -0.4144 & -0.3384 & 0.0000 & -0.2393 \\ 0.0328 & -0.3384 & -0.2393 & 0.0232 & 0.0000\end{array}\right]$,

$\vartheta=\left[\begin{array}{ccccc}0.0000 & -0.8592 & -2.8285 & -2.1845 & -2.4329 \\ -6.6453 & 0.0000 & -2.8049 & -3.2735 & -2.0397 \\ -5.8099 & -2.7779 & 0.0000 & -2.9972 & -1.4858 \\ -5.3477 & -2.5294 & -1.5764 & 0.0000 & -2.5145 \\ -5.5507 & -3.3285 & -3.3236 & -3.7103 & 0.0000\end{array}\right]$.

Step 5: get the overall dominance degree $\delta\left(A_{i}\right)$ of the alternative $A_{i}$. The overall dominance degree $\delta\left(A_{i}\right)$ can be obtained according to formula (25) and is listed in Table 4. 
TABLE 1: Data information.

\begin{tabular}{cccccccc}
\hline & $C_{1}$ & $C_{2}$ & $C_{3}$ & $C_{4}$ & $C_{5}$ & $C_{6}$ & $C_{7}$ \\
\hline$A_{1}$ & 162.2 & 0.05 & 0.04 & 0.46 & 3.2 & $\left\{\mathrm{~S}_{3}(0.3), \mathrm{S}_{2}(0.5), \mathrm{S}_{-1}(0.2)\right\}$ & $\left\{\mathrm{S}_{3}(0.9), \mathrm{S}_{1}(0.1)\right\}$ \\
$A_{2}$ & 280.1 & 0.09 & 0.03 & 0.22 & 2.4 & $\left\{\mathrm{~S}_{3}(0.5), \mathrm{S}_{2}(0.4)\right\}$ & $\left\{\mathrm{S}_{3}(0.7), \mathrm{S}_{1}(0.3)\right\}$ \\
$A_{3}$ & 338.0 & 0.10 & 0.01 & 0.19 & 5.3 & $\left\{\mathrm{~S}_{2}(0.7), \mathrm{S}_{1}(0.2), \mathrm{S}_{-2}(0.1)\right\}$ & $\left\{\mathrm{S}_{3}(0.8), \mathrm{S}_{1}(0.1), \mathrm{S}_{0}(0.1)\right\}$ \\
$A_{4}$ & 186.7 & 0.07 & 0.01 & 0.22 & 7.3 & $\left\{\mathrm{~S}_{2}(0.6), \mathrm{S}_{1}(0.3), \mathrm{S}_{0}(0.1)\right\}$ & $\left\{\mathrm{S}_{3}(0.8), \mathrm{S}_{2}(0.1), \mathrm{S}_{1}(0.1)\right\}$ \\
$A_{5}$ & 226.5 & 0.11 & 0.03 & 0.12 & 5.2 & $\left\{\mathrm{~S}_{2}(0.5), \mathrm{S}_{1}(0.3), \mathrm{S}_{-1}(0.2)\right\}$ & $\left\{\mathrm{S}_{3}(0.6), \mathrm{S}_{2}(0.4)\right\}$ \\
\hline
\end{tabular}

TABLE 2: Normalized data information.

\begin{tabular}{cccccccc}
\hline & $C_{1}$ & $C_{2}$ & $C_{3}$ & $C_{4}$ & $C_{5}$ & $C_{6}$ & $C_{7}$ \\
\hline$A_{1}$ & 0.4799 & 1.0000 & 1.0000 & 1.0000 & 0.4384 & $\left\{\mathrm{~S}_{-3}(0.3), \mathrm{S}_{-2}(0.5), \mathrm{S}_{1}(0.2)\right\}$ & $\left\{\mathrm{S}_{-3}(0), \mathrm{S}_{-3}(0.9), \mathrm{S}_{-1}(0.1)\right\}$ \\
$A_{2}$ & 0.8287 & 0.5556 & 0.7500 & 0.4783 & 0.3288 & $\left\{\mathrm{~S}_{-3}(0), \mathrm{S}_{-3}(0.5556), \mathrm{S}_{-2}(0.4444)\right\}$ & $\left\{\mathrm{S}_{-3}(0), \mathrm{S}_{-3}(0.7), \mathrm{S}_{-1}(0.3)\right\}$ \\
$A_{3}$ & 1.0000 & 0.5000 & 0.2500 & 0.4130 & 0.7260 & $\left\{\mathrm{~S}_{-2}(0.7), \mathrm{S}_{-1}(0.2), \mathrm{S}_{2}(0.1)\right\}$ & $\left\{\mathrm{S}_{-3}(0.8), \mathrm{S}_{-1}(0.1), \mathrm{S}_{0}(0.1)\right\}$ \\
$A_{4}$ & 0.5524 & 0.7143 & 0.2500 & 0.4783 & 1.0000 & $\left\{\mathrm{~S}_{-2}(0.6), \mathrm{S}_{-1}(0.3), \mathrm{S}_{0}(0.1)\right\}$ & $\left\{\mathrm{S}_{-3}(0.8), \mathrm{S}_{-2}(0.1), \mathrm{S}_{-1}(0.1)\right\}$ \\
$A_{5}$ & 0.6701 & 0.4545 & 0.7500 & 0.2609 & 0.7123 & $\left\{\mathrm{~S}_{-2}(0.5), \mathrm{S}_{-1}(0.3), \mathrm{S}_{1}(0.2)\right\}$ & $\left\{\mathrm{S}_{-3}(0), \mathrm{S}_{-3}(0.6), \mathrm{S}_{-2}(0.4)\right\}$ \\
\hline
\end{tabular}

TABLE 3: The relative weight.

\begin{tabular}{lccccccc}
\hline & $C_{1}$ & $C_{2}$ & $C_{3}$ & $C_{4}$ & $C_{5}$ & $C_{6}$ & $C_{7}$ \\
\hline Weight & 0.2173 & 0.1452 & 0.2084 & 0.0809 & 0.1755 & 0.0757 & 0.0971 \\
Relative weight & 1.0000 & 0.6681 & 0.9594 & 0.3724 & 0.8077 & 0.3485 & 0.4467 \\
\hline
\end{tabular}

TABLE 4: Overall dominance degree for all alternatives.

\begin{tabular}{lclc}
\hline Alternative & The overall dominance degree & $\delta\left(A_{i}\right)$ & Ranking \\
\hline$A_{1}$ & -8.3051 & 1.0000 & 1 \\
$A_{2}$ & -14.7634 & 0.1511 & 4 \\
$A_{3}$ & -13.0708 & 0.3736 & 3 \\
$A_{4}$ & -11.9680 & 0.5185 & 2 \\
$A_{5}$ & -15.9131 & 0.0000 & 5 \\
\hline
\end{tabular}

Step 6: rank all the alternatives. The ranking of all the alternatives can be obtained according to the overall dominance degree $\delta\left(A_{i}\right)$ and is listed in Table 4 . The larger the value of $\delta\left(A_{i}\right)$ is, the better the alternative is. We can see the best alternative is $A_{1}$, and the ranking is $A_{1}>A_{4}>A_{3}>A_{2}>A_{5}$. That is to say, the most serious city of the urban COVID-19 epidemic is $A_{1}$. Obviously, our proposed hybrid TODIM method is feasible and can get a good decision result because our proposed hybrid method can not only involve multiple criteria or attributes about qualitative and quantitative aspects, but also express their judgments and reflect different probability degrees of all the possible linguistic information or preference information.

5.3. Sensitivity Analysis. In order to further verify the validity and effectiveness of the hybrid TODIM method with crisp number and PLTS, sensitivity analysis of the parameter $\theta$ is carried out for urban epidemic situation evaluation. In the hybrid TODIM method, the dominance degree $\phi_{j}\left(A_{i}, A_{t}\right)$ of the alternative $A_{i}$ over the alternative
$A_{t}$ with respect to the criterion $C_{j}$ is determined based on the parameter $\theta$, which is the attenuation factor of the losses. So, it is very necessary and important to analyze the sensitivity of the parameter $\theta$ according to the different values for each simulation, and the ranking results are presented in Table 5.

From Table 5, the ranking orders of alternatives are consistent when the parameter $\theta$ takes different values from 0.1 to 2 , which indicates the validity, effectiveness, and stability of the proposed hybrid TODIM method with the changes of the attenuation parameter $\theta$. And our proposed hybrid TODIM method involves multiple criteria or attributes about qualitative and quantitative aspects for improving the accuracy of evaluation results; besides, our proposed hybrid TODIM method can not only express their judgments or linguistic preference with multiple linguistic terms, but also reflect different probability degrees of all the possible linguistic information or preference information. In addition, the concept of entropy and probability linguistic entropy is applied to induce hybrid criteria weight information. 


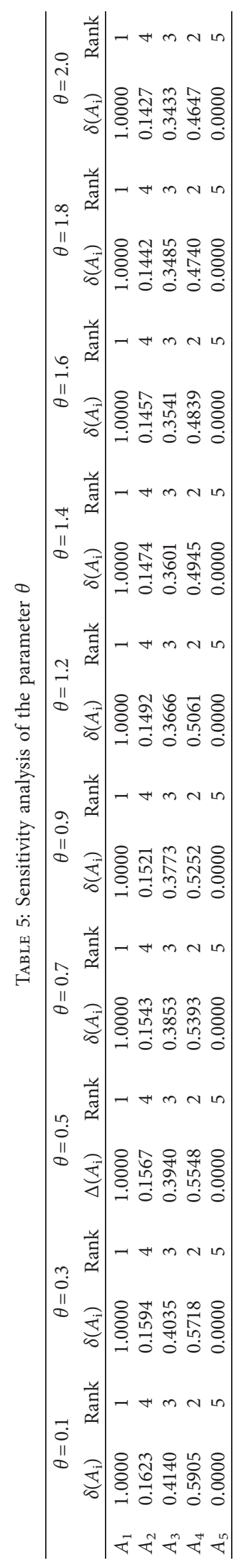




\section{Conclusions}

The frequent occurrence of emergency medical incidents has caused a great deal of property losses and a lot of casualties [17]. Emergency medical management and epidemic situation evaluation has a complex evolutionary process, and emergency decision-making is very difficult to deal with the traditional decision theory. So, in this paper, based on the TODIM method considering the psychology and behavior of the DMs and PLTS expressing preference information of DMs, a hybrid TODIM method with crisp number and PLTS is proposed for aiming to assist DMs to evaluate the severity of urban COVID-19 epidemic situation involved in emergency medical management. Five main advantages of this paper are summarized as follows: firstly, the extended hybrid TODIM method is first proposed for epidemic situation evaluation during a complex humanitarian crisis environment. Secondly, in this hybrid TODIM method, multiple criteria or attributes about qualitative and quantitative aspects are involved, considering the complexity of emergency decision-making process. Thirdly, the proposed hybrid TODIM method can not only express their judgments or linguistic preference with multiple linguistic terms, but also reflect different importance degrees or probability degrees of all the possible linguistic information or preference information. Fourthly, the concept of entropy and probability linguistic entropy is applied to induce hybrid criteria weight information, which has the advantages of simple calculation and clear concept. Finally, sensitivity analysis of the parameter, which considers the psychology factors and cognitive behavior of the DMs, is further carried out on a case study to demonstrate the validity, effectiveness, and stability of the proposed hybrid method for urban epidemic situation evaluation.

The limitations of this work are summarized as follows: epidemic situation evaluation has a complex evolutionary process. Through literature research, based on the complexity of emergency decision-making process, the TODIM method is still not well studied and applied for emergency medical decision-making, although it has done a lot of outreach and extension. So, in this paper, the extended hybrid TODIM method with crisp number and PLTS is first proposed for epidemic situation evaluation. However, the sample size of the data is relatively small.

In future work, new and effective evaluation methods will be further researched and developed by comparative analysis to effectively respond to emergency medical emergencies during a complex humanitarian crisis environment and the crisis response strategies will also be the focus of our research. In addition, big data analysis techniques will also be further developed to reduce the impact of small sample sizes on research results.

\section{Data Availability}

The data used to support the findings of this study are included within the article.

\section{Conflicts of Interest}

The authors declare that they have no conflicts of interest.

\section{Acknowledgments}

This research has been partially supported by Grants from Fund for Less Developed Regions of the National Natural Science Foundation of China (\#71761014), the State Key Program of National Natural Science Foundation of China (\#71532007), and Postdoctoral Science Foundation Project of China (\#2016M592683).

\section{References}

[1] W. Wu, G. Kou, and Y. Peng, "Group decision-making using improved multi-criteria decision making methods for credit risk analysis," Filomat, vol. 30, no. 15, pp. 4135-4150, 2016.

[2] D. Kahneman and A. Tversky, "Prospect theory: an analysis of decision under risk," Econometrica, vol. 47, no. 2, pp. 263-291, 1979.

[3] L. Gomes and M. Lima, "TODIM: basics and application to multi-criteria ranking of projects with environmental impacts," Foundations of Computing and Decision Sciences, vol. 16, no. 4, pp. 113-127, 1991.

[4] P. Liu and X. You, "Probabilistic linguistic TODIM approach for multiple attribute decision-making," Granular Computing, vol. 2, no. 4, pp. 333-342, 2017.

[5] P. Liu and F. Teng, "An extended TODIM method for multiple attribute group decision making based on intuitionistic uncertain linguistic variables," Journal of Intelligent \& Fuzzy Systems, vol. 29, no. 2, pp. 701-711, 2015.

[6] P. Rani, D. Jain, and D. S. Hooda, "Extension of intuitionistic fuzzy TODIM technique for multi-criteria decision making method based on shapley weighted divergence measure," Granular Computing, vol. 4, no. 3, pp. 407-420, 2019.

[7] R. Davoudabadi, S. M. Mousavi, and V. Mohagheghi, "A new last aggregation method of multi-attributes group decision making based on concepts of TODIM, WASPAS and TOPSIS under interval-valued intuitionistic fuzzy uncertainty," Knowledge and Information Systems, vol. 62, no. 4, pp. 1371-1391, 2020.

[8] S. P. Singh and P. Singh, "A hybrid decision support model using axiomatic fuzzy set theory in AHP and TOPSIS for multicriteria route selection," Complex \& Intelligent Systems, vol. 4, no. 2, pp. 133-143, 2018.

[9] M. Tavana, F. Zandi, and M. N. Katehakis, "A hybrid fuzzy group ANP-TOPSIS framework for assessment of e-government readiness from a CiRM perspective," Information \& Management, vol. 50, no. 7, pp. 383-397, 2013.

[10] Q. Pang, H. Wang, and Z. Xu, "Probabilistic linguistic term sets in multi-attribute group decision making," Information Sciences, vol. 369, pp. 128-143, 2016.

[11] S. H. Kim and B. S. Ahn, "Group decision-making procedure considering preference strength under incomplete information," Computer \& Operations Research, vol. 24, pp. 11011112, 1997.

[12] Z. Xu and N. Zhao, "Information fusion for intuitionistic fuzzy decision making: an overview," Information Fusion, vol. 28, pp. 10-23, 2016.

[13] W. S. Wu, Z. S. Xu, G. Kou, and Y. Shi, "Decision-making support for the evaluation of clustering algorithms based on MCDM," Complexity, Article ID 9602526, 17 pages, 2020.

[14] W. Wu, G. Kou, Y. Peng, and D. Ergu, "Improved ahp-group decision making for investment strategy selection," Technological and Economic Development of Economy, vol. 18, no. 2, pp. 299-316, 2012. 
[15] G. Kou and W. Wu, "Multi-criteria decision analysis for emergency medical service assessment," Annals of Operations Research, vol. 223, no. 1, pp. 239-254, 2014.

[16] Z. Xu and H. Wang, "On the syntax and semantics of virtual linguistic terms for information fusion in decision making," Information Fusion, vol. 34, pp. 43-48, 2017.

[17] W. S. Wu and Y. Peng, "Extension of grey relational analysis for facilitating group consensus to oil spill emergency management," Annals of Operations Research, vol. 238, no. 1-2, pp. 615-635, 2016.

[18] L. L. Pullum and A. Ramanathan, "Oak ridge biosurveillance toolkit: scalable machine learning for public health surveillance," in proceedings of the 2014 IEEE 4th International Conference on Computational Advances in Bio and Medical Sciences (ICCABS), Miami, FL, USA, 2014.

[19] H. E. Wang, J. P. Donnelly, D. Barton, and J. L. Jarvis, "Assessing advanced airway management performance in a national cohort of emergency medical services agencies," Annals of Emergency Medicine, vol. 71, no. 5, pp. 597-607, 2018.

[20] J. L. Temple, D. Hostler, C. Martin-Gill et al., "Systematic review and meta-analysis of the effects of caffeine in fatigued shift workers: implications for emergency medical services personnel," Prehospital Emergency Care, vol. 22, no. sup1, pp. 37-46, 2018.

[21] E. H. Lazzara, J. R. Keebler, M. L. Shuffler, B. Patzer, D. C. Smith, and P. Misasi, "Considerations for multiteam systems in emergency medical services," Journal of Patient Safety, vol. 15, no. 2, pp. 150-153, 2019.

[22] Y. Peng, G. Kou, G. Wang, W. Wu, and Y. Shi, "Ensemble of software defect predictors: an AHP-based evaluation method," International Journal of Information Technology \& Decision Making, vol. 10, no. 01, pp. 187-206, 2011.

[23] W. S. Wu and G. Kou, "A group consensus model for evaluating real estate investment alternatives," Financial Innovation, vol. 2, no. 1, p. 8, 2016.

[24] R. R. Kumar, S. Mishra, and C. Kumar, "Prioritizing the solution of cloud service selection using integrated MCDM methods under Fuzzy environment," Journal of Supercomputing, vol. 4, pp. 1-31, 2017.

[25] X. B. Mao, N. Shang, R. Zhang, and M. Wu, "TODIM method based on probabilistic linguistic term sets and its application in enterprise performance evaluation of strategic emerging industries," Operations Research and Fuzziology, vol. 8, no. 4, pp. 151-160, 2018.

[26] G. Wei, “TODIM method for picture fuzzy multiple attribute decision making," Informatica, vol. 29, no. 3, pp. 555-566, 2018.

[27] Y. Zhang, Z. Xu, and H. Liao, "Water security evaluation based on the TODIM method with probabilistic linguistic term sets," Soft Computing, vol. 23, no. 15, pp. 6215-6230, 2019.

[28] Z.-P. Fan, X. Zhang, F.-D. Chen, and Y. Liu, "Extended TODIM method for hybrid multiple attribute decision making problems," Knowledge-Based Systems, vol. 42, pp. 40-48, 2013.

[29] P. Liu and F. Teng, "An extended TODIM method for multiple attribute group decision-making based on 2-dimension uncertain linguistic variable," Complexity, vol. 21, no. 5, pp. 20-30, 2016.

[30] Z. Xu, "Deviation measures of linguistic preference relations in group decision making," Omega, vol. 33, no. 3, pp. 249-254, 2005.
[31] Z. S. Xu, Linguistic Decision Making: Theory and Methods, Springer, Berlin, Germany, 2012.

[32] B. Farhadinia, "Determination of entropy measures for the ordinal scale-based linguistic models," Information Sciences, vol. 369, pp. 63-79, 2016.

[33] M. W. Lin and Z. S. Xu, "Probabilistic linguistic distance measures and their applications in multi-criteria group decision making," in Soft Computing Applications for Group Decision-making and Consensus Modeling, Studies in Fuzziness and Soft Computing, M. Collan and J. Kacprzyk, Eds., vol. 357, pp. 411-440, Springer, Cham, Switzerland, 2018. 